\title{
BONLAC: A Combinatorial Proteomic Technique to Measure Stimulus-induced Translational Profiles in Brain Slices
}

\author{
Heather Bowling ${ }^{1, *}$, Aditi Bhattacharya ${ }^{1, *}$, Guoan Zhang ${ }^{2}$, \\ Joseph Z. Lebowitz ${ }^{1}$, Danyal Alam ${ }^{1}$, Peter T. Smith ${ }^{3}$, Kent \\ Kirshenbaum $^{3}$, Thomas A. Neubert ${ }^{2}$, Christine Vogel ${ }^{4}$, Moses V. Chao ${ }^{5 \#}$, \\ Eric Klann ${ }^{1 \#}$ \\ ${ }^{1}$ Center for Neural Science, New York University, New York, NY \\ ${ }^{2}$ Kimmel Center for Biology and Medicine at the Skirball Institute and \\ Department of Biochemistry and Molecular Pharmacology, \\ New York University School of Medicine \\ ${ }^{3}$ Department of Chemistry, New York University \\ ${ }^{4}$ Department of Biology, Center for Genomics and Systems Biology, \\ New York University \\ ${ }^{5}$ Departments of Cell Biology, Physiology, and Neuroscience andPsychiatry, \\ Kimmel Center for Biology and Medicine at the Skirballlnstitute, New York \\ University School of Medicine \\ ${ }^{*}$, "\#Contributed equally to the work
}

Corresponding authors: Eric Klann and Moses V. Chao 


\begin{abstract}
Stimulus-triggered protein synthesis is critical for brain health and function. However, due to technical hurdles, de novo neuronal translation is predominantly studied in cultured cells, whereas electrophysiological and circuit analyses often are performed in brain slices. The different properties of these two experimental systems create an information gap about stimulus-induced alterations in the expression of new proteins in mature circuits. To address this, we adapted two existing techniques, BONCAT and SILAC, to a combined proteomic technique, BONLAC, for use in acute adult hippocampal slices. Using BDNF-induced protein synthesis as a proof of concept, we found alterations in expression of proteins involved in neurotransmission, trafficking, and cation binding that differed from those found in a similar screen in cultured neurons. Our results indicate important differences between cultured neurons and slices, and suggest that BONLAC could be used to dissect proteomic changes underlying synaptic events in adult circuits.
\end{abstract}

Keywords: BONLAC, BONCAT, SILAC, de novo proteomics, BDNF, hippocampal slice synaptic plasticity, protein synthesis 


\section{Introduction}

De novo protein synthesis is a requirement for long-lasting changes in neuronal function and the consolidation of memory ${ }^{1,2}$. Because neurons are polarized and have upwards of 10,000 synapses, on-demand mRNA translation occurs both in soma and dendrites, which results in rapid and stimulus-specific protein expression ${ }^{3}$. Translational homeostasis therefore represents a major point of control for protein abundance ${ }^{4}$, and is critical for proper brain function.

Interestingly, our knowledge of neuronal translational control is predominantly derived from dissociated primary cultures and immortalized cell lines. In contrast, most information on synaptic plasticity, such as long term potentiation (LTP) and long-term depression (LTD), are derived from acutely prepared brain slices. These two systems are intrinsically disparate. For example, cultures are a monolayer of largely homogenous, disaggregated individual neurons that form stochastic connections as opposed to the multi-layer and genetically-encoded spatial organization that exists in slices. In addition, cultured neurons and mature brain slices represent different developmental time points, and brain slices contain glia and endothelial cells, which influence the final circuit response of a slice and is absent in neuronal cultures. These fundamental differences between slices and neuronal culture likely influence the overall proteomic and signaling profiles in response to various types of stimulation. Although the adult hippocampal slice has been used extensively to characterize multiple forms of synaptic plasticity, to datethere are limited examples of large-scale unbiased study of proteomic response to chemical and electrical triggers in a slice, highlighting a gap in our current knowledge. 
Among the available profiling techniques, shotgun proteomics has emerged as the method of choice given its high-throughput approach ${ }^{5}$. Recently, bioorthogonalnoncanonical amino acid tagging (BONCAT, Supplementary Figure $1 A^{6}$ ) and stable isotope labeling by amino acid in cell culture (SILAC, Supplementary Figure $1 \mathrm{~B}^{7,8}$ ) wereintroduced to selectively isolate and quantify de novo proteins synthesized within a specified temporal window. BONCAT involves the tagging of nascent peptides with a methionine analog, which then is detected using a tagged-alkyne probe by a Huisgen chemistry reaction called cycloadditon ${ }^{9}$. SILAC employs isotopically-labeled, differentially weighted (heavy or medium) arginine and lysine that are incorporated into newly synthesized proteins to provide a mass spectrometry (MS)profile within the time frame of labeling ${ }^{5}$. These techniques have been used in comparative proteomic analysis of protein synthesis in several cell culture systems, including cell lines that have higher protein synthesis rates than non-dividing cells ${ }^{10-12}$. However, neither of these approacheseither singly or in combinationhave been modified to measure the expression of newly synthesized proteins in an intact brain circuit.

We sought to adapt both BONCAT and SILAC to a multi-layered slice preparation to follow ongoing proteomic changes. As a standard for comparison, we used the well-studied neurotrophic factor,Brain-derived neurotrophicfactor (BDNF), which has been shown to trigger neuronal protein synthesis, including local translation in cultured neurons ${ }^{13-15}$. In slices, BDNF is mostly utilized to facilitate protein-synthesis dependent LTP ${ }^{16-20}$. We began by modifying the existing predominantly culture-based BONCAT and fluorescent non-canonical amino acid tagging (FUNCAT)-based protocols to study the response of a multi-layered slice to changes in protein synthesis by western 
blotting and immunofluorescence respectively. We then performed quantitative proteomics to identify and quantify the newly synthesized proteins using combinatorial approach of SILAC and BONCAT, which we have termed BONLAC, and then screened for changes in protein abundance of selected candidates. We finally compared the slice data to a similar BONLAC screen in cultured neurons, where we found that though candidates in slices and cultured neurons diverged appreciably, the cultured neuron data was consistent with previous reports suggesting inherent differences in proteomic response between the two systems. In summary, we demonstrate that BONLAC is a relatively easy and fast approach that is applicable to monitoring proteomic changes in response to a range of stimuli in intact brain slices.

\section{Results}

BONCAT and FUNCAT detect increases in protein synthesis in multiple layers of the hippocampal slice.

BONCAT has been shown to detect neuronal translation in cultures previously ${ }^{15}$, but required standardization for application in slices. This involved determining the incubation parameters of the methionine analog azido-homoalanine (AHA) and characterizing the specificity of the AHA-alkyne signal in a multi-layered tissue preparation. The standard incubation and manipulation medium for acute brain slices is artificial cerebrospinal fluid (ACSF), which does not supplement amino acids to the tissue as is done in culture media. Thus,slices probably retain an internal store of amino acids for several hours after dissection, the dynamics of which could modify the incorporation of the AHA into the available pool of amino acids.In addition, mammalian 
brains contain endogenous proteins that are post-translationally modified to contain biotin, which may interfere with signal detection. We tested a range of AHA concentrations from $0.5 \mathrm{mM}$ to $3 \mathrm{mM}$, based on previous reports, and incubated slices with AHA for 1-5 hours after recovery (see Methods). We found an optimal dose and timeframefor the application of BONCAT to slices at $1 \mathrm{mM}$ AHA incubated for three hours (see Supplementary Figure S2A). To test the specificity of the BONCAT signal, we stimulated protein synthesis by treating slices with $1 \mu \mathrm{M}$ Insulin for tenminutesand pre-incubated slices with $40 \mu \mathrm{M}$ anisomycin to abrogate translation. We observed robust increase in AHA signal in the insulin-treated slices,and almost entirely abrogated signalin the anisomycin-treated samples (Supplementary Figure S2B). We noted the presence of two bands that appear consistently on all western blots and likely are signal of previously synthesized proteins complexed with biotin that did not change with AHA dose or any treatment (Supplementary Figure S2A-B, white arrows). We also adapted the BONCAT-based biotin-avidin precipitation protocol to isolate biotin-alkyne labeled nascent proteins from these lysates and found a complete washout of signal in the anisomycin-treated lanes (Supplementary Figure S2B, avidinpulldown). These results indicate that BONCAT is sensitive to dynamic alterations in translation in slices, such as insulin-induced protein synthesis, and that the AHA-alkyne-biotin detection method is largely capturing newly synthesized proteins within a given temporal window.

After establishing that BONCAT could detect short-term changes in protein synthesis, we then sought to examine the proteomic response to brain-derived neutrophic factor (BDNF), a plasticity-inducing growth factor that has been shown tofacilitate $L T P^{16-20}$. We incubated hippocampal slices with 25ng/ml BDNF for onehour 
to detect elevated translation by BONCAT. After treatment of slices with BDNF for one hour we noted a significant increase in AHA incorporation in hippocampal lysates (Figure 1B-C) that was correlated with increased phosphorylation of BDNF receptor tyrosine kinase receptor B (TrkB) (Supplementary Figure S2A). Thus, BONCATis able to detectspecific changes in protein synthesis driven by a biological stimulus by western blot in slices.

We proceeded to determine the spatial and regional pattern of the translational response to BDNF treatment in hippocampal slices. For this we independently derived a protocol similar to Dieck et al ., ${ }^{15}$ for use of FUNCAT to brain slices, where AHA is detected using an alkynetagged to Alexa 488 andsubsequent fluorescence imaging. BDNF treatment of one hour resulted in an increase in fluorescence in area CA1 and the dentate gyrus (DG) compared to control slices, which was present in both the soma and the dendrites of the neurons (Figure 1D-E). In addition, there was a greater BDNFinduced change in new protein synthesis in the DG compared to area CA1(Figure 1DE). Because slices are multi-layered, it was unclear whether the BDNF-mediated increase in translation was either restricted to specific layers or was uniform throughout the slice. As electrophysiological recordings are best obtained from internal layers of a slice, it was important to establish the spatial distribution of the proteomic response. For this, we re-sectioned individual $400 \mu \mathrm{m}$ BDNF-treated slices into thinner $40 \mu \mathrm{m}$ slices in serial order and analyzed every third slice (Supplementary Figure S2B-C). We noted that elevated levels of protein synthesis induced by BDNF occurred throughout the entire $400 \mu \mathrm{m}$ slice with the maximal fluorescence in the middle of theslice (Supplementary Figure S2D). Notably, non-neuronal components of the slice also 
contributed to the overall FUNCAT signal (Supplementary Figure S2C, yellow arrows) suggesting an increase in non-neuronal protein synthesis in response to BDNF. The experiment also demonstrated that the AHA was transported and incorporated throughout the entire slice within a few hours of labeling, suggesting that FUNCAT is effective in detecting changes in the translational landscape across the entire thickness of hippocampal slices. 
A

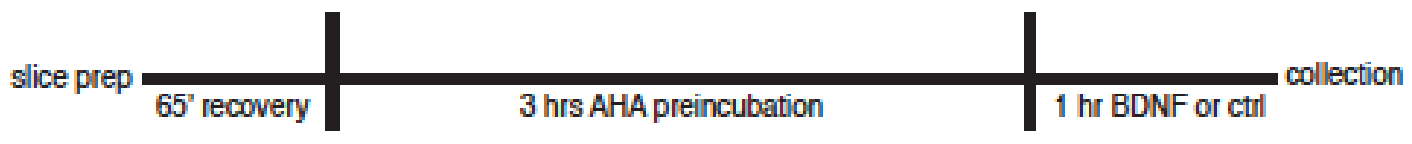

B
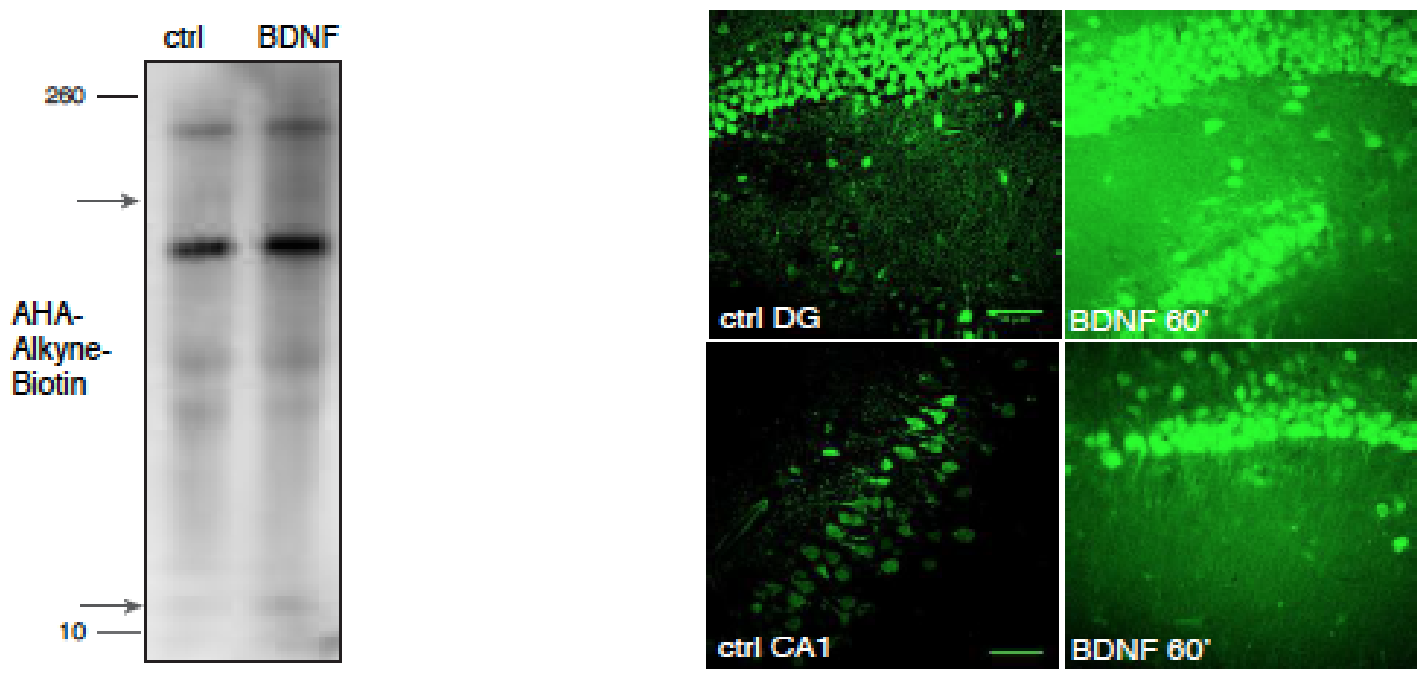

C

E

DG

CA1
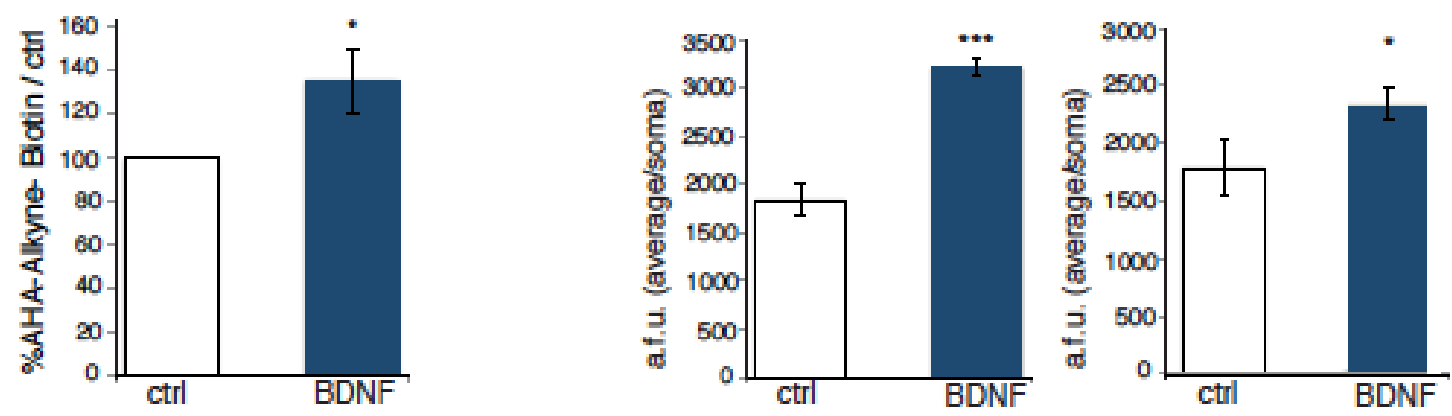

Figure 1: Characterization of translational dynamics in intact hippocampal slices in response to BDNF. A) Timeline of treatment of acute adult mouse hippocampal slices. B) Representative western blot of BONCAT-labeled proteins in lysates from hippocampal slices treated with and without BDNF. Arrows denote examples where bands are noticeably increased with BDNF treatment. C) Bar graphs demonstrating percent upregulation in mRNA translation as measured by BONCAT in BDNF-treated slices. $n=7$ with 5-6 slices per condition. Statistical analyses using a two-tailed Student's 
$t$-test, $p=0.03$. Average \pm SEM shown for all graphs.D) Representative images of the dentate gyrus (DG) and area CA1 of hippocampal slices with and without 1 hour of BDNF treatment. Incorporation of AHA as detected by FUNCAT with alkyne-Alexa488

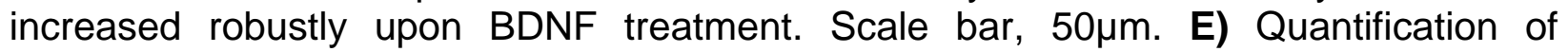
increase in AHA-alkyne-Alexa488 signal in arbitrary fluorescent units (a.f.u) in BDNFtreated slices. 20 cell somas in each slice were randomly chosen from the bottom, middle and top thirds of the image from corresponding DIC images of somas in the CA1 and the Dentate Gyrus (DG) of the hippocampus as noted and the intensity was measured in ImageJ. Statistical analyses using the Kolmogorov-Smirnov test (control vs BDNF); $p=0.00001$ for DG and $p=0.0009$ for CA1. $N=20$ somas per slice, 10 slices measured for BDNF and 6 for control from three independent experiments. Average \pm SEM shown.

\section{Quantificationof BDNF-induced proteomic changes with BONLAC- Mass}

\section{Spectrometry}

Having standardized the general regime of incubating slices with AHA and detecting a specific signal, we next asked whether we could determine the identities of the proteins whose synthesis is regulated by BDNF within onehour of application. A major challenge for proteomic studies in slices is the isolation of denovo peptides synthesized within a particular temporal window and distinguishing them from the abundant background of pre-existing peptides. BONCAT and pulsed SILAC have been used together to circumvent this limitation in cell culture systems, and have been previously termed BONLAC or QuaNCAT ${ }^{10,11,12,20}$. However, pulsed SILAC alone in cultured neurons requires incubation for times that far exceed the normal lifespan of an acute slice preparation ${ }^{23}$, leading to the need for quick enrichment for nascent peptides. We adapted and optimized this combinatorial technique for use inbrain slices using the protocol shown in Figure 2A-B. Slices were incubated with AHA and either heavy- or medium-labeled arginine and lysine for four hours and then exposed to BDNF for 1 hour. We chose an extended incubation time for the weighted amino acids to facilitate greater 
incorporation of these probes into slices. Pilot experiments carried out with incubation times of less than four hours yielded poor MS coverage (data not shown).

Lysates from the vehicle and treated slices were combined and then underwent cycloaddition and AHA-tagged peptides were isolated on an alkyne-resin (BONCAT), followed by proteolytic digestion and LC/MS-MS. Relative signals of heavy versus medium labeled peptides were used to quantify the abundance of a particular protein in the sample (SILAC). We carried out three biologically independent replicates of the BONLAC experiment, each containing slices from three mice per condition.Although we could confirm that AHA penetrated the slices at the timepoint used for BONLAC (Supplementary Figure S2C-D), there is no fluorescent corollary available for SILAC that could be used. Therefore,we hypothesized that the kinetics of SILAC incorporation into tissue would follow a similar trend and that proteins labeled with AHA also would be labeled with SILAC. To confirm that co-labeling occurred, weanalyzed the SILAC content of the BONCAT-enriched proteins after cycloaddition, enrichment, and analysis by MS. The histogram shown in Figure $2 \mathrm{C}$ depicts the frequency of proteins detected at a light (no SILAC), medium and heavier masses (SILAC-labelled), with the blue bar indicating proteins whose masses indicate that they were SILAC labeled. We noted that the majority of proteins enriched by BONCAT contained a SILAC label, confirming that all three amino acid substitutes (AHA, heavy/medium Arginine and heavy/medium Lysine) were incorporated into the same proteins.

We obtained a total of2159 identifiable proteinsthree times, with 2875 detected at least twice across the three runs (complete raw data available at ftp://MSV000078919@massive.ucsd.edu). Average fold change and intensities then 
were measured for each protein across the biological replicates, normalized, and plotted to yield the distribution shown in Figure 2D. Our MS analyses revealed that the majority of proteins did not differ between treatment groups, which is shown by the cluster of proteins shown in black around $\log _{2} 0$ in the plot in Figure 2D. The relative similarity in synthesis in the majority of proteins suggests that the increase in overall protein synthesis observed by immunofluorescence and western blotting in Figure 1 may bedue to changes in the relative abundance of specific proteins (Figure 2D, complete list Supplementary Table S1). 
A

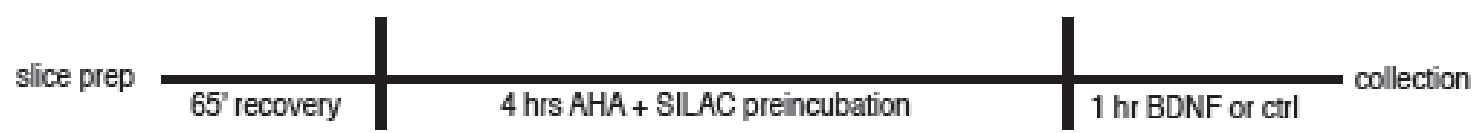

B

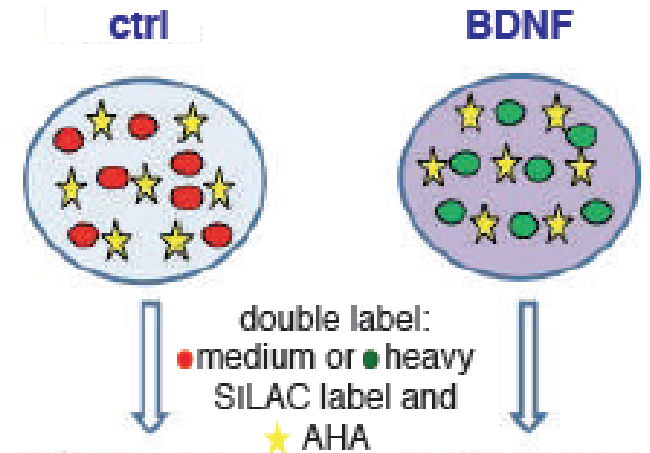

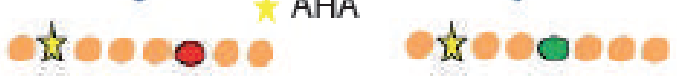

Combine lysates \&

isolate nascent

proteins

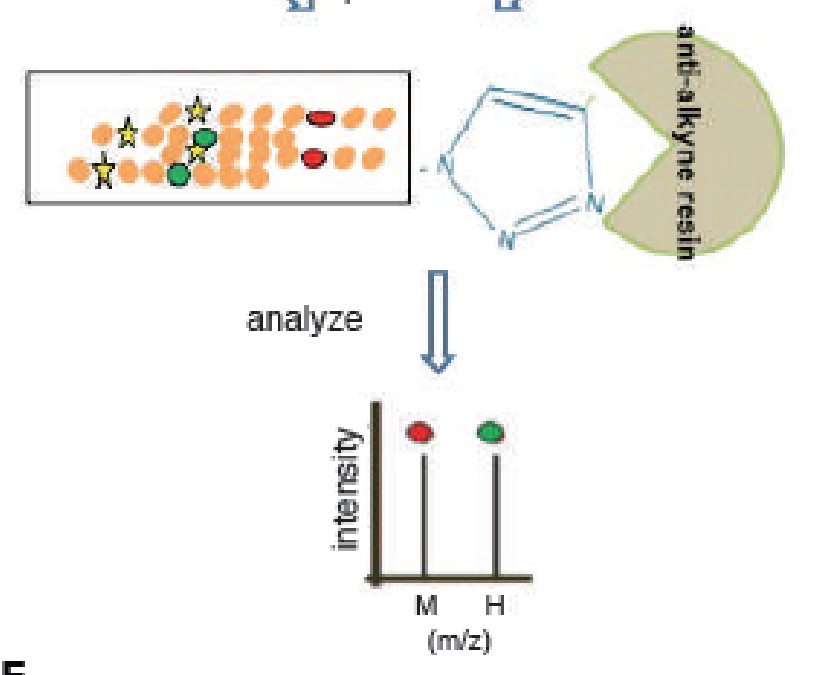

E

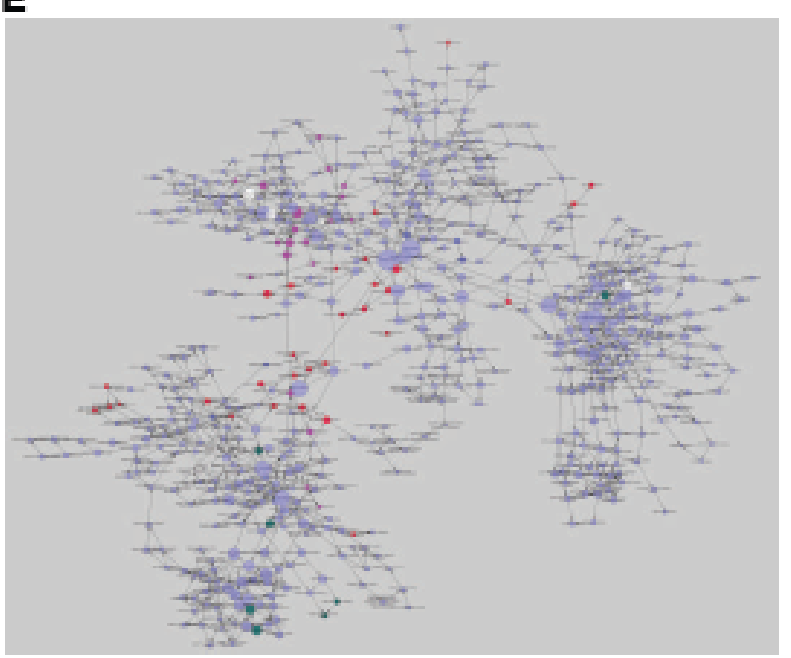

C

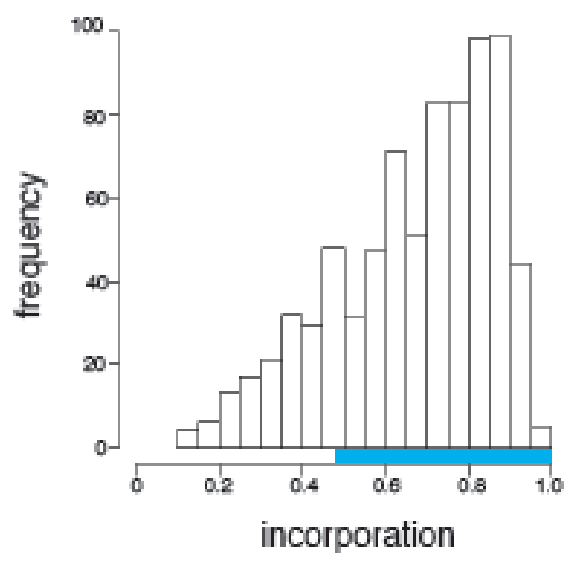

D

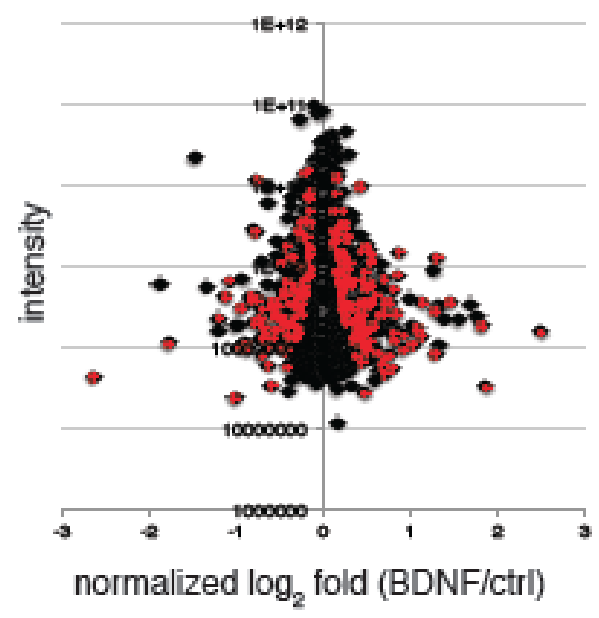

synaptic release / synapse

neuronal development /

synaptic plasticity

cytoskeleton

signaling

other 
Figure 2: BONLAC analyses of hippocampal slices stimulated with BDNF.

A) Timeline of BONLAC experiments. B) Representative workflow of a BONLAC experiment. Stars represent AHA, red and green ovals represent medium and heavy labeled amino acids (lysine and arginine). Lysates were combined after treatment and underwent the same nascent protein extraction and mass spectrometry analysis to provide direct quantitation and comparison of control and BDNF treated groups. C) Histogram of SILAC incorporation of BONCAT-enriched proteins. Blue bar denotes SILAC-labeled proteins. There were more SILAC-labeled proteins than unlabeled proteins. D) Fold change vs intensity distribution plot of all detected proteins in BDNFtreated versus untreated conditions in black. Y-axis indicated the average intensity of the protein and $x$-axis indicates the average normalized $\log _{2}$ fold change (BDNF/ctrl). Proteins whose expression increased with BDNF are on the positive side of $x$-axis, while those that decreased are 0 the negative side of the $x$-axis. Overlay with red scatter plot depicts candidate proteins that were consistently altered using cut-offs derived from C-score rank score algorithm. $\mathrm{n}=3 \mathrm{MS}$ runs involving 3 mice per condition per run. E) Functional clustering represented graphically using Cytoscape. Candidates for BDNF-induced proteins were queried. Groups shown had an association of $p<0.05$. Groups were generally categorized into the listed clusters. Red=synaptic proteins, magenta=synaptic plasticity proteins, white $=$ cytoskeleton proteins, green $=$ signaling proteins and purple denotes protein clusters that are not otherwise specified. There were a notable number of clusters of proteins related to synapses that were enriched after BDNF treatment.

We next analyzed the relative abundance of proteins that were differentially affected by BDNF versus vehicle. To ensure that specific subsets of proteins were not selected artificially, we determined the average fold-change for each protein with no cutoffs and manually classified them into one of ten categories based on their primary role in translation, signaling, transcription, trafficking, protein folding, synaptic function, cytoskeleton, metabolism, and degradation (Supplementary Figure S3). The protein hits fell into a variety of functional bins, with no apparent bias suggesting that a wide variety of proteins were detected with BONLAC and that the profiling approach in slices were representative of a global proteome.

Because verifiable protein expression changes in a neurophysiological context tend to be small relative to those detected in dividing cells (i.e. much smaller than the 
frequently used $\geq 1.5$ fold metric) ${ }^{22,23}$, the standard customary quantitationusing False Discovery Rate (FDR)corrected fold-change cutoffs likely underrepresent biologically meaningful proteomic changes. Therefore, we refined prevailing software and analysis techniques to add a consistency metric to rank-order proteins according to their small, but consistent expression changes. This combined consistency score (abbreviated henceforth as C-score for easy reference), encompassed two criteria: 1) the combined significance of the average SILAC fold change with the intensity measurement of a given protein (Significance_B FDR from Max Quant's Perseus package) and 2) the consistency of expression changes across replicates (see Methods).Simply put, this analysis included proteins that were either upregulated or downregulated consistently and removed any proteins that changed in opposing directions across replicates. Cscoremaximized the inclusion of a true-positive set of proteins known to change expression in response to BDNF and are the subset of proteins shown in red in Figure 2D.

To further refine this candidate protein list, we performed a protein function cluster analysis with gene ontology (GO) using DAVID ${ }^{24}$, and selected candidate subsets that were significantly overrepresented in BDNF dataset as compared to control (FDR of DAVID cluster $<0.05$, Supplementary Table S2). High enrichment was found in metabolic, cation-binding, calcium-related, and glycoprotein/secretion protein classes (Table 1). We also noted that non-neuronal processes, such as hemopoeisis, were also enriched categories in the GO analysis (Supplementary Table S2), although to a lesser extent, further suggesting the potential contribution of non-neuronal cells to the BDNF response in slices. 
Table 1: Cation-binding, calcium and secreted proteins are enriched after BDNFtreatment. Summary of the proteins in the top GO functional clusters. Candidates were clustered by DAVID, and those clusters with high FDRs and functional relevance were selected.All values have been rounded to 2 decimal places. The Average Fold Change was calculated by MaxQuant, The Enrichment Score and Group FDR was calculated by DAVID and Significance was calculated by Perseus.

\begin{tabular}{|c|c|c|}
\hline Gene Name & Average Log2 Fold Change & Significance \\
\hline Cation Binding & Enrichment Score: 3.87 & Group FDR: $1.2 \mathrm{E}-3$ \\
\hline Crp & -2.64 & 0.00 \\
\hline Ehd3 & 0.15 & 0.24 \\
\hline Gatad2b & -0.12 & 0.29 \\
\hline Kcnip4 & 1.31 & 0.00 \\
\hline Lasp1 & 0.25 & 0.05 \\
\hline Necab1 & -0.89 & 0.01 \\
\hline Necab2 & 1.28 & 0.00 \\
\hline Ndufa8 & 0.26 & 0.04 \\
\hline Rasgrp1 & -1.12 & 0.00 \\
\hline S100a16 & 0.26 & 0.16 \\
\hline Sgip1 & 0.21 & 0.25 \\
\hline Acat1 & 0.49 & 0.00 \\
\hline Adi1 & 0.35 & 0.16 \\
\hline Alb & -1.77 & 0.00 \\
\hline Anxa11 & 0.21 & 0.09 \\
\hline Arsa & 0.91 & 0.02 \\
\hline Cdh5 & 0.20 & 0.27 \\
\hline Cacna2d1 & 0.81 & 0.02 \\
\hline Cacng8 & -1.20 & 0.00 \\
\hline Cat & 0.32 & 0.01 \\
\hline Cpsf3 & 0.48 & 0.24 \\
\hline Crip1 & 0.43 & 0.22 \\
\hline Crip2 & -0.44 & 0.08 \\
\hline
\end{tabular}




\begin{tabular}{|c|c|c|}
\hline Egr3 & -0.29 & 0.15 \\
\hline Enpp2 & 0.55 & 0.00 \\
\hline Eps15I1 & 0.22 & 0.22 \\
\hline Glo1 & 0.29 & 0.11 \\
\hline Hebp1 & 0.46 & 0.01 \\
\hline $\mathrm{Hba}$ & -0.75 & 0.00 \\
\hline Idh2 & 0.35 & 0.16 \\
\hline Mt2 & 0.33 & 0.01 \\
\hline Myt1l & -0.33 & 0.20 \\
\hline Nbr1 & -0.37 & 0.14 \\
\hline Pam & 0.19 & 0.30 \\
\hline Tpt1 & 0.28 & 0.03 \\
\hline Rps27a & -0.17 & 0.13 \\
\hline Pkm2 & 0.60 & 0.02 \\
\hline Me1 & 1.09 & 0.01 \\
\hline Rps29 & 0.54 & 0.03 \\
\hline Pdcd6 & 0.47 & 0.18 \\
\hline Prkcsh & 0.27 & 0.28 \\
\hline Ppm1a & 0.25 & 0.17 \\
\hline Ppp1cb & 0.13 & 0.29 \\
\hline Ppp1ca & 1.13 & 0.00 \\
\hline Ppp3r1 & -0.26 & 0.02 \\
\hline Ppp3cb & 0.70 & 0.05 \\
\hline Dnaja4 & -0.94 & 0.00 \\
\hline Snrpc & 0.22 & 0.09 \\
\hline Slc12a2 & 0.32 & 0.09 \\
\hline Smap1 & 0.57 & 0.00 \\
\hline Sod2 & -0.21 & 0.30 \\
\hline Syp & -0.23 & 0.24 \\
\hline Syt3 & -0.42 & 0.22 \\
\hline Syt5 & -0.22 & 0.26 \\
\hline Syt7 & 1.29 & 0.00 \\
\hline Timm9 & 0.80 & 0.00 \\
\hline Uqcrfs1 & 0.19 & 0.29 \\
\hline Uqcrc2 & 0.53 & 0.00 \\
\hline Vcan & -0.46 & 0.19 \\
\hline Vsnl1 & -0.19 & 0.09 \\
\hline Zc3hav1 & 0.78 & 0.03 \\
\hline
\end{tabular}




\begin{tabular}{|c|c|c|}
\hline Zfp36l1 & 0.24 & 0.19 \\
\hline Zcchc18 & -0.18 & 0.11 \\
\hline Calcium & Enrichment Score 2.22 & Group FDR 1.5E-3 \\
\hline Crp & -2.64 & 0.00 \\
\hline Ehd3 & 0.15 & 0.24 \\
\hline Kcnip4 & 1.31 & 0.00 \\
\hline Necab1 & -0.89 & 0.01 \\
\hline Necab2 & 1.28 & 0.00 \\
\hline Rasgrp1 & -1.12 & 0.00 \\
\hline S100a16 & 0.26 & 0.16 \\
\hline Anxa11 & 0.21 & 0.09 \\
\hline Arsa & 0.91 & 0.02 \\
\hline Cdh5 & 0.20 & 0.27 \\
\hline Cacna2d1 & 0.81 & 0.02 \\
\hline Cacng8 & -1.20 & 0.00 \\
\hline Eps15l1 & 0.22 & 0.22 \\
\hline Tpt1 & 0.28 & 0.03 \\
\hline Pdcd6 & 0.47 & 0.18 \\
\hline Prkcsh & 0.27 & 0.28 \\
\hline Ppp3r1 & -0.26 & 0.02 \\
\hline Syp & -0.23 & 0.24 \\
\hline Syt3 & -0.42 & 0.22 \\
\hline Syt5 & -0.22 & 0.26 \\
\hline Syt7 & 1.29 & 0.00 \\
\hline Vcan & -0.46 & 0.19 \\
\hline Vsnl1 & -0.19 & 0.09 \\
\hline Glycoprotein/Secreted & Enrichment Score 2.53 & Group FDR 2.8E-2 \\
\hline Pddc1 & -0.59 & 0.13 \\
\hline Arsa & 0.91 & 0.02 \\
\hline BDNF & -0.39 & 0.05 \\
\hline Cdh5 & 0.20 & 0.27 \\
\hline Cacna2d1 & 0.81 & 0.02 \\
\hline Ctsd & 0.24 & 0.06 \\
\hline Col4a2 & 0.46 & 0.07 \\
\hline
\end{tabular}




\begin{tabular}{|l|r|r|} 
Enpp2 & 0.55 & 0.00 \\
\hline Et14 & 0.22 & 0.23 \\
\hline H2-D1 & 0.18 & 0.15 \\
\hline Icam5 & -0.44 & 0.08 \\
\hline Nrn1 & 1.88 & 0.00 \\
\hline Nefl & -0.19 & 0.09 \\
\hline Nptn & -0.42 & 0.00 \\
\hline Omg & 0.55 & 0.12 \\
\hline Pam & 0.19 & 0.30 \\
\hline Hspg2 & 0.42 & 0.23 \\
\hline Prdx1 & 0.23 & 0.07 \\
\hline Phyhip & -0.32 & 0.10 \\
\hline Plat & 0.21 & 0.11 \\
\hline Plau & 0.36 & 0.15 \\
\hline Prkcsh & 0.27 & 0.28 \\
\hline Serpinh1 & -0.79 & 0.02 \\
\hline Slc12a2 & 0.32 & 0.09 \\
\hline Slc6a17 & -0.44 & 0.08 \\
\hline Sort1 & -0.28 & 0.27 \\
\hline S1pr1 & -1.01 & 0.01 \\
\hline Syp & -0.23 & 0.24 \\
\hline Vcan & -0.46 & 0.19 \\
\hline
\end{tabular}

BDNFupregulates mediators of synaptic transmission and signaling in hippocampal slices, the synthesis of which is dependent on multiple signaling pathways.

To complement the functional clustering data obtained from DAVID, we used BiNGOGO software ${ }^{25}$ to map the interactions of the candidate proteins, again finding that a substantial number of the most consistently hits were involved in synaptic release 
(red, Figure 2E), supporting the previous DAVID analysis (Table 1). BDNF is thought to mediate LTP via pre- and post-synaptic mechanisms, and hence for validation, we elected to focus on those candidates that play a role in synaptic transmission and were highly represented in the $G O$ analysis ${ }^{16-20}$. We selected three candidates: sortilin (Sort1), synaptotagmin 7 (Syt7), and voltage-dependent calcium channel subunit alpha2/delta-1 (Cacna2d1) to proceed with our validation as they are involved in synaptic release, and their expression has not been previously reported to be regulated by BDNF in slices. Sort1 is a known intracellular interactor of neurotrophins including pro-BDNF, and is involved in the activity-driven release of pro- and mature BDNF $^{26}$. Syt7, a calcium-binding presynaptic protein, is a known sensor for synaptic vesicle replenishment and mediates a switch to slow asynchronous transmitter release in neurons, but this specific isoform has not been studied in context of BDNF function ${ }^{27,28}$. Cacna2d1 is an auxiliary subunit of voltage-activated calcium channels and is implicated in regulation of synaptic transmission and neuronal excitability ${ }^{29,30}$. Our BONLAC MS results predicted an increase in Syt7 (average $\log _{2}$ fold change: 1.29) and Cacna2d1(0.81)levels with decreased Sort1(- 0.28) levels in slices post-BDNF treatment. We found a significant increase in Syt7 and Cacna2d1, and a significant decrease in Sort1 levels with BDNF treatment in the total lysates of acute hippocampal slices treated with BDNF for 1 hour(Figure3A-C). To confirm that these changes were the result of new protein synthesis, we used streptavidin precipitationto isolate nascent peptides from BDNF-treated hippocampal lysates and probed for Sort1, Syt7 and Cacna2d1.In the avidin-affinity-precipitated fractions, we confirmed an enrichment of Cacna2d1 and Syt7 in BDNF-treated samples, and a simultaneous depletion of Sort1 
(Supplementary Figure S5A). These results support the notion that BDNF treatment alters the total and newly synthesized levels of proteins involved in synaptic release and calcium signaling in adult hippocampal slices. Furthermore, the validation of these candidates identified by BONLACunderscoresthe reproducibility of BONLAC in mature circuits and its ability to identify proteins with altered translation in response to stimuli.

Identifying and quantifying proteomic candidates that change with synaptic events in slices is useful in the investigation of synaptic plasticity and translational control, however, the ability to understand how this translational control is exerted is also valuable. To address how translation is regulated in response to BDNF in slices, weasked how general translation and specific synthesis of the validated candidates were regulated by BDNF-dependentsignaling pathways ${ }^{31-33}$. To establish the relative contributions of different signaling pathways, we first asked whether the majority of these short-term proteomic changes were due to either transcription (ActinomycinD) or translation (Anisomycin). We pre-labeled slices with AHA and introduced the inhibitorsone hour before BDNF treatment to measure their effect on BDNF-induced protein synthesis.Our results suggest that translation, and not transcription, is the main contributor to the early BDNF proteomic response (Supplementary Figure S5B). We then determined the contributions of specific signaling pathways to the BDNF-induced increase in protein synthesis. The mammalian target of rapamycin complex 1 (mTORC1) and extracellular-signal regulated kinase 1/2 (ERK1/2) pathways have been shown to be required for BDNF-induced increases in de novo protein synthesis ${ }^{18,34}$. Hence, we pretreated slices with either the MEK-ERK inhibitor PD098059 or the mTORC1 inhibitor rapamycin. We also conducted experiments where we applied 
tetrodotoxin, which predominantly blocksevoked neurotransmitter release,to determine whether interruption of normalsynaptic transmission would affect BDNF-induced translation in theslices. We found that PD098059 and rapamycin both attenuated BDNF-induced increases in protein synthesis(Figure 3A, D-E). When PD089059, rapamycin, and tetrodotoxin were applied together, the decrease in BDNF-induced protein synthesis was additive (Figure 3D-E). Taken together, these results suggest that the de novo translation in slices following BDNF treatment involves multiple downstream signaling pathways that act concordantly to regulate translation.

We next examined how tetrodoxin, rapamycin and PD098059 treatment affected the levels of Sort1, Syt7 and Cacna2d1 in BDNF-treated hippocampal slices. Tetrodotoxin and rapamycin significantly reduced BDNF-induced increases in Cacna2d1 and increased Syt7 levels even further (Figure 3F-G). There was no effect of PD098059 on either Cacna2d1 or Syt7. Sort1 was decreased by BDNF administration, but its levels were slightly increased by PD098059 treatment (Figure 3F-G). These data suggest that although electrical activity, mTORC1 signaling, and ERK signaling contribute to general BDNF-induced translation, the regulation of specific mRNA transcripts is pathway-specific. 
A

$1 \mathrm{hr}$ inhibitors

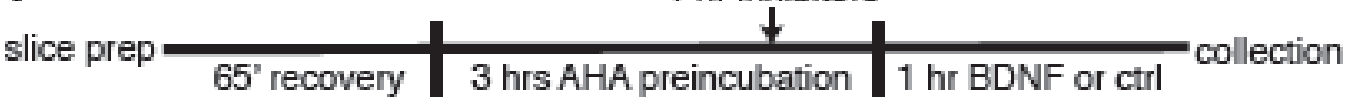

B
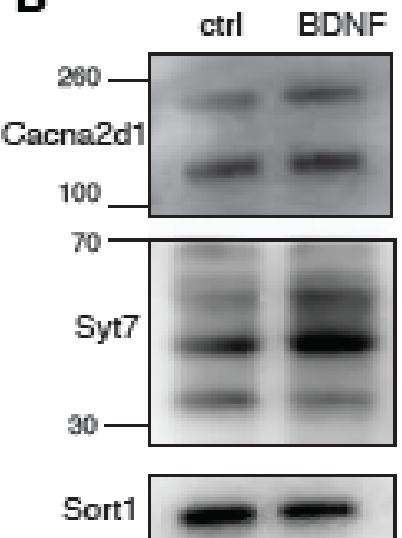

Actin

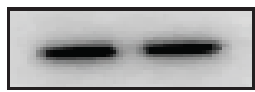

C
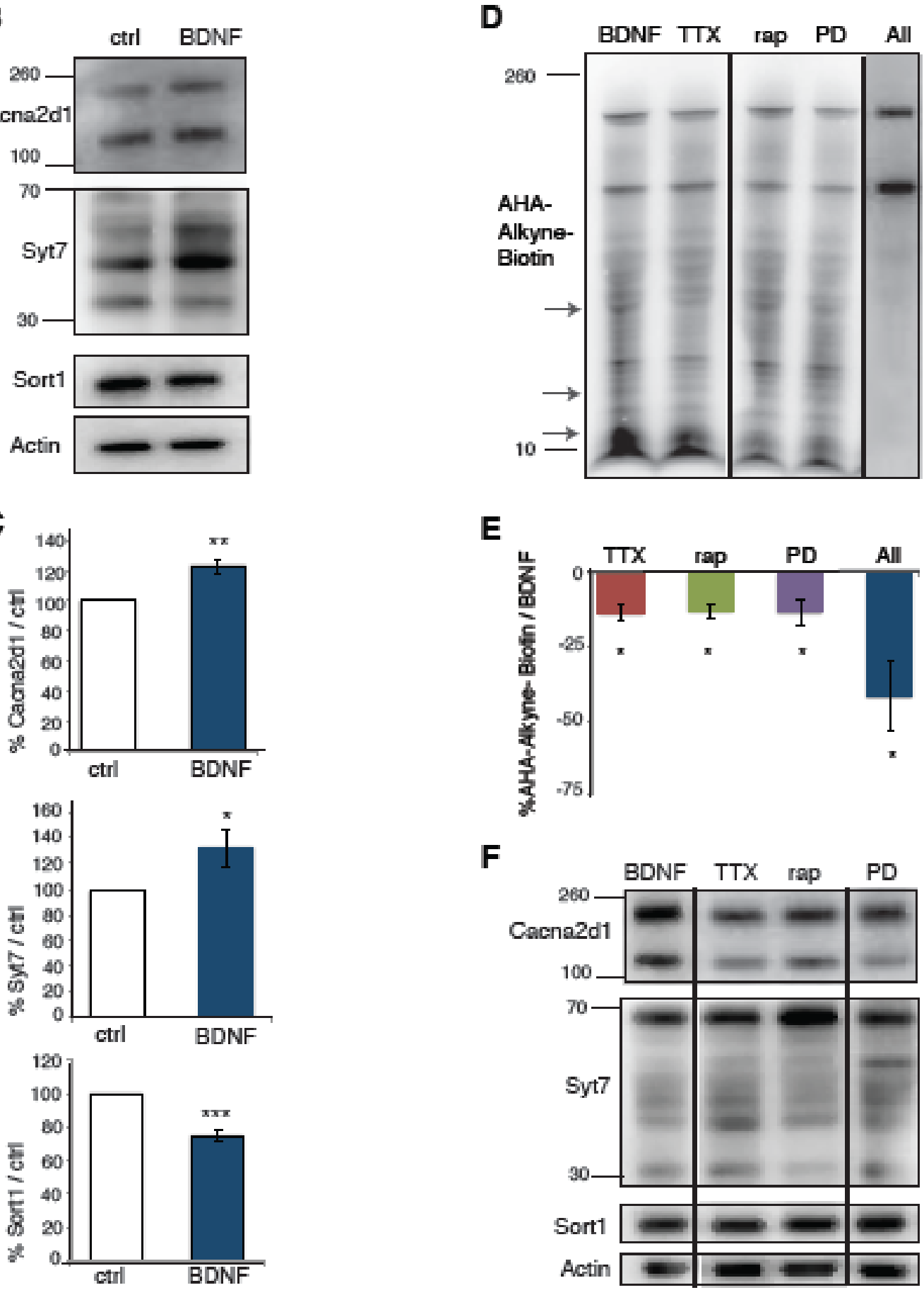

E

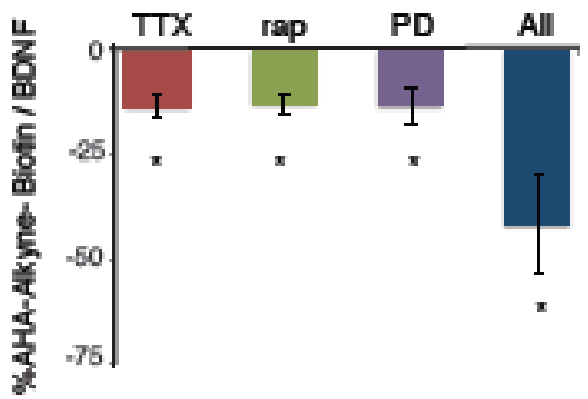

F

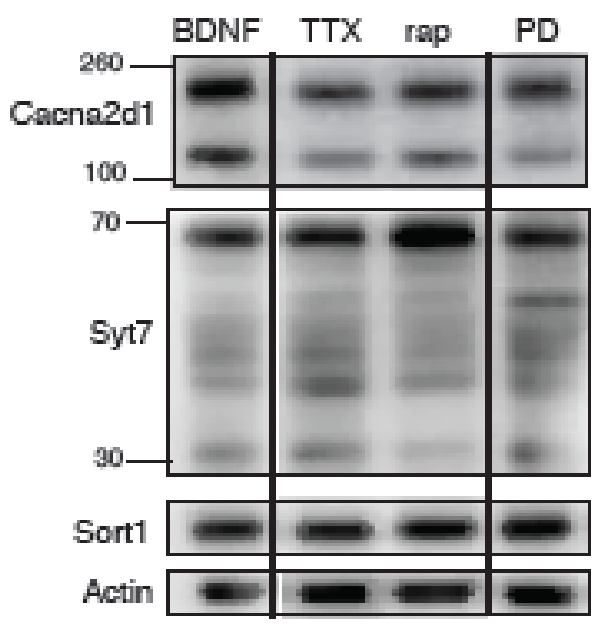

G
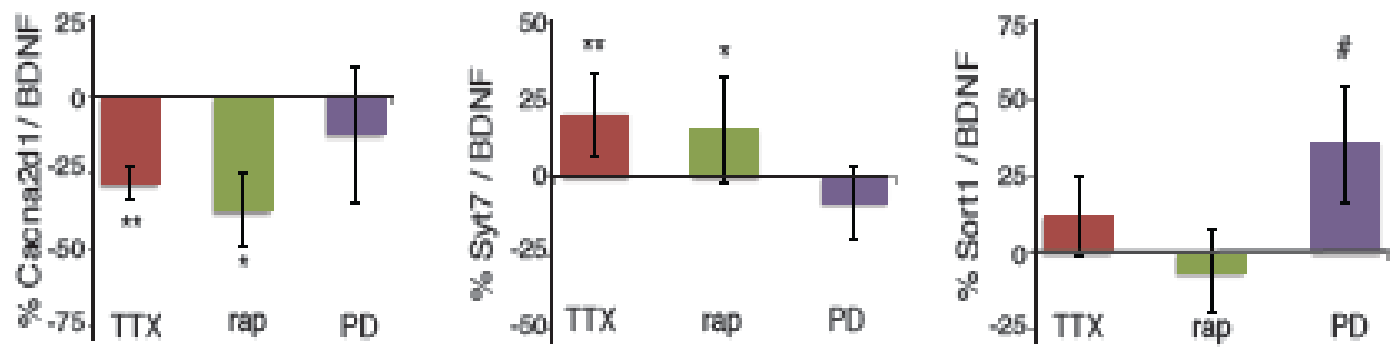
Figure 3: Validation of protein candidates identified by BONLAC and C-score dissection of signaling contributions that contribute to BDNF-induced alterations in protein synthesis. A) Timeline for treatment with inhibitors. B) Candidate proteins Cacna2d1, Syt7 and Sort1 were examined by western blotting following treatment with BDNF in total hippocampal lysates. C) Quantification of Cacna2d1, Syt7 and Sort1 western blots. There was a significant increase in Cacna2d1 and Syt7 following BDNF treatment. Control vs BDNF Cacna2d1 $n=3, p=0.01$, control vs BDNF Syt7 $N=6, p=$ 0.03. Sort1 was decreased following BDNF treatment. Control vs. BDNF Sort1 $N=3, p$ $=0.001$. These results were consistent with the predicted changes from mass spectrometry. D) Representative BONCAT western blot demonstrating the dependence of BDNF-induced translation on synaptic transmission (TTX), MEK-ERK (PD098059), mTORC1 (rap), or all combined (all). Inhibitors were added 1 hour before BDNF treatment to examine their effects on BDNF-induced protein synthesis. Arrows denote bands that clearly change with inhibitor treatment. E) Bar graphs representing percentage AHA-alkyne-biotin signal from slices treated with TTX, PD, and rapamycin slices (singly or combined) as compared to slices treated with BDNF alone. $n=3-4$ independent experiments, with 4-5 slices per condition per experiment. Statistical analyses using a two-tailed Student's $t$-test. $p=0.003$ (BDNF vs TTX-BDNF), $p=0.001$ (BDNF vs PD-BDNF), $p=0.01$ (BDNF vs Rap-BDNF) and $p=0.02$ (BDNF vs All-BDNF). F) Western blots of candidate proteins treated with BDNF, BDNF+TTX, BDNF+Rap, and BDNF+PD. G) Quantification of western blots. Cacna2d1 was reduced with TTX and Rap, but not PD. BDNF vs BDNF+TTX $N=4, p=0.01$, BDNF vsBDNF+Rap $n=4$, $p=0.04$. BDNF vs BDNF+PD $n=6, p=n s$. Syt7 was further increased with BDNF+TTX and BDNF+Rap treatment, but there was no effect of PD. BDNF vs TTX $n=4, p=0.002$, BDNF vsBDNF+Rap $n=3, p=0.03$, BDNF vs BDNF+PD $n=6, p=n s$. Sort1 was trend level increased in BDNF+PD, but not in the other paradigms. BDNF vs BDNF+TTX or BDNF+Rap $n=4, p=n s, B D N F$ vs. BDNF+PD, $n=6, p=0.09$. All graphs are shown as average \pm SEM.

\section{BDNF regulatesthe synthesis of different functional sets of proteins in slices}

\section{compared to cultured neurons}

To ensure that the changes observed in hippocampal slices were not artifacts of

BONLAC, it was important to compareour BONLAC screen with previously reported BDNFproteomic studies. Because the majority of these were performed in culture systems ${ }^{8,14}$ we opted to perform a comparison study in DIV 10 primary neuronal cultures. Previous studies in cultured neurons revealed that in contrast to cell lines, 5 
hours of treatment with pulsed SILAC was not sufficient to detect proteomic changes without enrichment ${ }^{23}$. DIV10 cultured neurons were exposed to either BDNF or vehicle for 2 hours. The number of days in culture was selected based on previous protocols ${ }^{8}$ and the two-hour labeling scheme was adapted from earlier BONLAC work in HEK cells ${ }^{12}$ (Figure 4A). After treatment we utilizedthe same MS and analytical workflow using Cscore to select consistently changed candidate proteins. The average fold-change was plotted with the average intensity across three biological replicates, with those that passed the C-score criteria shown in red (Figure 4B).We again noted that there was enrichment for SILAC-labeled proteins after BONLAC (Figure 4C, blue bar) andof the candidates that matched the consistency criteria, there was an enrichment of ribosomal,translation-related proteins, and immediate early genes,confirming data frommultiple previous studies(Figure 4D, full list Supplementary Table S4, ${ }^{14,}$ 18,35-37). This indicated that a different subset of proteins is altered in neuronal cultures compared to hippocampal slices (Figure 4D). We then asked to what extent the candidates identified by BONLAC and C-score in slicesalso were identified as candidates by the BONLAC screen in primary neuronal cultures. In total, there were 2393 proteins detected in common between cultured neurons and hippocampal slices. There were 212 candidates identified by C-score in slices (Supplementary Table S1), and 707 in primary cultured neurons (Supplementary Table S3). Though some proteins changed in the same direction between cultured neurons and slices, the majority of hippocampal slice c-score candidates were not conserved in cultured neurons (Table 2). Only 16 proteins were candidates and changed in the same direction in both slices and primary neurons, including Sort1 and Rpl36a (Figure 4E). The DAVID- GO analysis in 
cultured neurons also differed greatly from slices (Supplementary Table S4), with the primary group enriched being ribosomal proteins, as opposed to calcium and cation signaling in slices (Table 1).We have confirmed that BONLAC can also be used to detect and quantify short-term proteomic changes in neuronal culture. Taken together, our results indicate that BONLAC proteomic data obtained from cultures treated with BDNFare similar to data from previous reports. Moreover, these results imply that the candidate proteins we identified in slices treated with BDNF and their differences from previous reports in culture are not artifacts of the technique. 
A
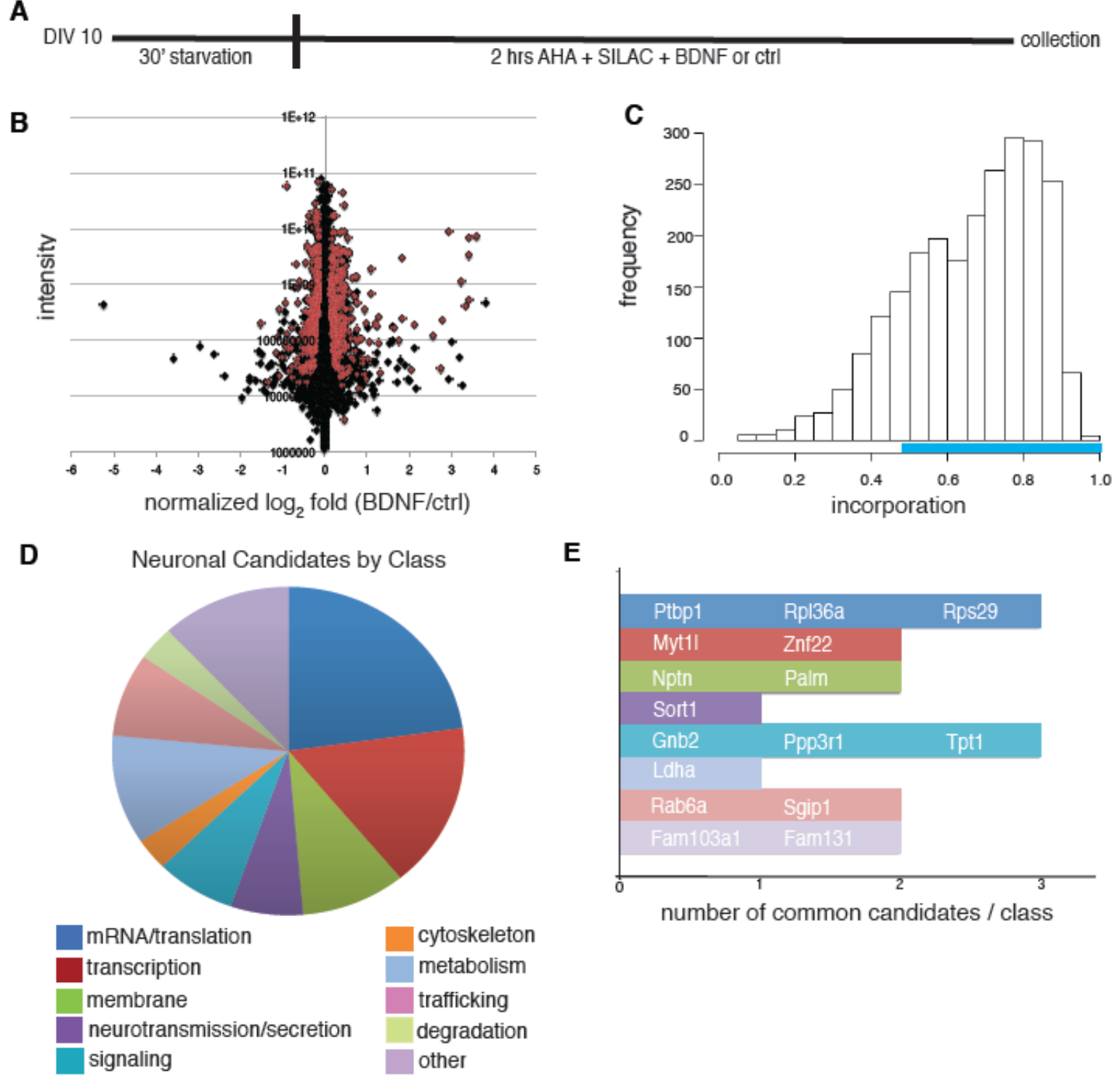

E

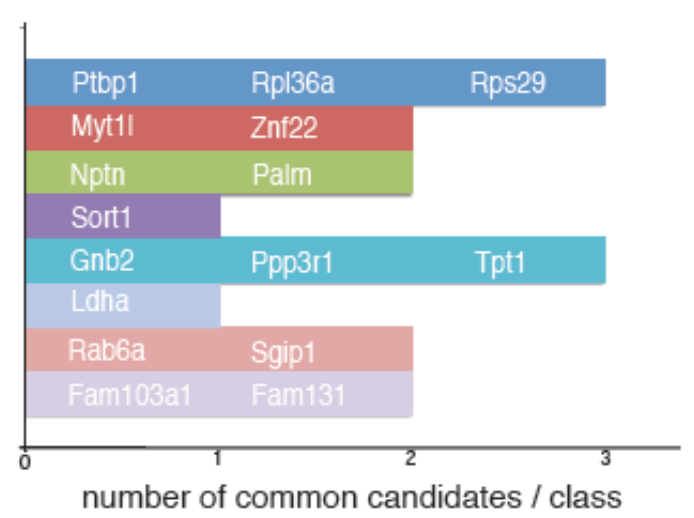

Figure 4: Comparison of BONLAC analyses in cultured neurons and hippocampal slices.

A) Timeline of BONLAC performed in DIV10 cultured neurons. B) Fold change vs intensity distribution plot of all detected proteins in BDNF-treated versus untreated conditions in black. $Y$-axis is the average intensity measured of a protein and the $\mathrm{X}$-axis is the average normalized $\log _{2}$ fold change (BDNF/ctrl). Overlay with red scatter plot depicts candidate proteins that were consistently altered using cut-offs derived from the c-score rank score algorithm. $n=3$ MS runs and 3 biological replicates.. C) Histogram of SILAC incorporation of BONCAT enriched proteins. Blue bar denotes SILAC-labeled proteins. There were more SILAC-labeled proteins than unlabeled proteins. D) Pie chart of functional classification of all protein hits from all runs of BONLAC screen divided by functional category. Protein IDs were queried on the UNIPROT database and functional classification based on broad categories listed underneath. E) The 16 candidates that were in both groups broken down by functional classification. 
Table 2. Comparison of hippocampal c-score hits with proteins measured in cortical neuron cultures.

C-score candidates from hippocampal slices were compared with proteins detected in the cultured neuron mass spectrometry screen. Though there were some proteins detected in common, few met C-score criteria in both groups.

\section{Category} Number

Total number C-score candidates slices

Total number C-score candidates cultured neurons

Total number consistent C-score candidates between

Opposite direction of cultured neurons

\section{Discussion}

Although the information generated from culture systems is invaluable, implicit in the neuroscience is the notion thatit represents the signaling and proteomic controlin mature, organized brains. The need for extrapolation instead of direct measurement is in part due to the current paucity of easy and inexpensive proteomic profiling techniques for complex brain tissue. Our data suggest that there are limits to extrapolation from culture to mature circuits, as we report minimal overlap in the proteomic response to the same stimulus. To circumvent these limitations,we have adapted, presented and evaluated a proteomic profiling method, BONLAC, specifically tailored to quantitatively 
assess de novo protein synthesis in acute brain slices within a short temporal window. The ability to directly quantifyde novo proteomic changes in a mature circuit is an important advance that addresses several of the limitations of previously available techniques.

All methods used here require small amounts of tissue, use reagents that are commercially available, and require no additional mice to be either designed and generated (riboTag) or maintained on a special diet (SILAM). BONLAC can potentially be used with any combination of mice genotypes, age and brain area.To our knowledge this is the first time that a BONCAT+SILAC combined technique has been used in a mature, multi-layer tissue system, addressing an existing technological hurdle and a known gap in our understanding of adult circuits. We elected to test BONLAC and the companion "C-score" analysis technique in response to a well-known neurotrophin, BDNF. In doing so, not only did we note some potential key differences between neurons in culture and hippocampal slices in terms of proteomic responses, but also differences in the protein classes that were regulated. Our validated results suggest that short-term BDNF treatment of adult hippocampal slices shifts the synaptic response by influencing proteins responsible for modulating synaptic release, which differs from results in neuronal cultures. Thus, BONLAC is a reliable method of detecting proteomic changes in more mature tissues, and is modifiable to a wide-range of applications.

Work from brain slices have used mRNA presence and localization as the most frequent metric to understand stimulus-driven changes in expression profiles. The limitation of microarrays, RNAseq, and RNA in situ work is the non-linear relationship between mRNA production and protein synthesis ${ }^{4}$. This non-linear relationship can 
occur due to regulation by microRNAs, degradation, changes in localization, and ribosomal stalling. Thus, predicting a final proteomic outcome by measuring either mRNA abundance or mRNAs associated with a ribosome are correlative and not equivalent to measuring nascent peptides.

Label free-proteomics does not distinguish between de novo and pre-existing peptides, whereas isotopic labeling MS approaches such as PSILAC alone are not able to identify enough newly synthesized proteins without a prior enrichment step in postmitotic cells and neurons.BONLACaddresses these concerns by allowing for a direct quantitative comparison of newly synthesized proteins on a short timescale. It had previously been used in human CD4+ cells ${ }^{10}$, HEK-TrkB cells ${ }^{12}$, and recently, cultured neurons ${ }^{38}$. However, it has never been adapted for use in three dimensional tissue systems. BONCAT alone has been used previously in acute adult hippocampal tissue 39; however, there was no additional label that allowed for a direct quantitative comparison by MS. In addition, BONLAC does not require either mice that have genetically manipulated suchas RiboTag or have been fedexotic amino acids such as SILAM, which are both expensive to generate and time-consuming to maintain. We therefore envisage BONLAC to be used in experiments where acutely prepared slices are used (ie, LTP and LTD) and for which the plasticity correlates are not entirely known. For a hypothetical example, with this technique it would be possible to identify which proteins are translated in response to theta-burst LTP and mTORC1 activation. Using the allied BONCAT and FUNCAT modalities, one can also quickly validate and localize the hits and shorten the turnaround time for gathering information on circuit specific responses. 
As important as it is to develop new methodologies for isolation newly synthesized peptides from slices, it is equally necessary to devise analytical approaches to quantify proteomic changes geared to the neural system. Existing MS analysis algorithms have been devised for cellular systems that show large-fold changes. In contrast mature, intact circuits tightly control their proteomic response and respond to stimuli with translational changes that are far more conservative in magnitude. This characteristic of adult brain tissue has been noted elsewhere previously and applying the traditional fold cut-offs could underestimate the proteomic response. It is also likely that small yet consistent changes in the levels of proteinssuch as an ion channel or a G-protein coupled receptor, would cause more significant and lasting impact on downstream signaling and ultimately, neuronal activity. This motivated us to adopt a new analysis schema in addition to the combinatorial detection that focuses on consistency rather than magnitude of the regulation, which can be erratic across several MS runs. Although we did observe that some candidate proteins showed consistently large-fold changes, most of our consistent hits were in a range of $10-30 \%$ change (Supplementary Table S1). Our downstream GO analyses also supported the validity of these hits, as the candidate proteins formed significantly enriched clusters (Supplementary Table S2), suggesting that they may have a common functional link. In addition, we also uncovered protein candidates that were not neuronal in origin, again supporting the inclusive nature of the translational landscape uncovered by BONLAC.

A major challenge in working with acutely prepared brain slices is their heterogeneous composition and relatively short life-span. Therefore it was important to standardize the labeling time for AHA and SILAC probes, such that enough signal could 
be obtained and slice health was not compromised. Because BONCAT and FUNCAT rely on signal amplification steps for western blotting and fluorescence imaging, respectively, we could optimize a shorter incubation period for these applications. We did not incorporate either the PD-10 column or the organic extraction step recommended in BONCAT protocols ${ }^{6}$ or Click-It reaction kit manual owing to the high lipid content in brain tissue which bind proteins in the lysate and decrease labeled peptide yield by almost 50\%. To circumvent this issue, we used dialysis for avidin precipitations and a different, specific protein isolation Click-It kit (see Methods). For BONLAC we were constrained to adopt a longer incubation time in order to accumulate sufficient amount of labeled material for MS analysis. Most importantly, we established the specific nature of the AHA-alkyne signal since most of it could be abrogated by preincubating slices with the protein synthesis inhibitoranisomycin. Although our standardization of BONCAT for slices provided a framework for further studies, our FUNCAT data uncovered novel dynamics of protein synthesis in slices. For example, although it is well known that area CA1 versus the dentate gyrus differ in circuit composition and functional output, it has not been clearly demonstrated that this correlates with differing short-term translation rates. Further, our serial FUNCAT study showed how responses to stimuli differ from the slice exterior to interior, which is consistent with the strongest LTP signal being recorded from the middle of the slice. Because the amount of material required to do these experiments is comparable to those used in routine western blot and immunohistochemical experiments, it is likely that future studies using these adapted BONCAT and FUNCAT methods could uncover more nuances of translation responses of a multilayered brain slice. 
Apart from characterizing the new improvement of the BONLAC combinatorial technique, our results also shed light on BDNF-mediated protein synthesis in hippocampal slices. It should be noted that for three candidate proteins, we observed not only altered de novo synthesis (affinity precipitation), but also altered expression in the total lysates. The increased expression of Cacna2d1 and Syt7 isclearly due to de novo protein synthesis, whereas the downregulation of Sort1 could be due to changes in either its degradation orthe involvement of other forms of regulation such as that by miRNAs. Our data implies that BDNF tempers the response of the hippocampus by altering the expression of proteins mediatingneuronal secretion and neurotransmitter release including Syt7, Cacna2d1, and Sort1 (Table 1). Although there is previous indirect evidence for the involvement of such proteins in BDNF-induced plasticity changes ${ }^{27,40-42}$, our results provide the first concrete evidence. Although more evidence is required to conclude that BDNF treatment can alter these specificsecretion and neurotransmitterrelease properties, thesefindings suggest a role for proteinsynthesisdependent changes in the expression of synaptic proteins in shaping the neuronal response to BDNF.

Another interesting finding was that, in the one-hour window of exposure to BDNF, the majority of translation was via pre-existing mRNA, rather than new mRNAs via transcription. This is consistent with previous data showing that immediate early gene expression in brain slices does not occur within this duration of BDNF exposure 18,40. The results with separate application of mTORC1 and MEK-ERK inhibitors indicated that both signaling pathways are involved in the elevated translation in response to BDNF. Although there are several reports of BDNF-induced synaptic 
release in neurons, our tetrodotoxin data suggest that this is important for upregulating protein synthesis in circuits as well. Interestingly, the larger inhibition obtained by the combination of allthree inhibitors indicates that signaling pathways are likely redundant and synergistically promote protein synthesis in response to BDNF.

Finally, our comparison of BDNF-induced proteomic changes in slices versus neurons revealed that the majority of the protein candidates altered were strikingly different. Some of the changes in candidate protein abundance suggested by the neuronal culture screen had been reported previously and/or validated, supporting the findings of the BONLAC screen 5,18, 35,36. The difference between the proteomic candidates identified in neuronal cultures and those in slices are likely not due to the difference in timeframe (one-hour BDNF treatment versus two-hours)as Liao et $\mathrm{al}^{14}$ measured changes after 30 minutes of BDNF treatment. The differences also are not likely due to differences in species(mouse versus rat species) as some of the previously reported candidates, such as Arc, have been reported to be altered in response to BDNF in both species(Supplementary Table S3) ${ }^{43}$.The response of neurons in culture can include excitation and BDNF release with the immediate addition of BDNF, whereas the changes in release in a slice could be more spatially and temporally regulated. Hippocampal slices and cultured neurons are distinct developmentally, which also could shape the differences in their de novo proteomic responses to BDNF.An additional factor in slices is the heterogeneity of non-neuronal cells that also contain TrkB receptors, which could mount their own response and are capable of influencing the signaling of neurons ${ }^{44}$. For these reasons, though invaluable, the information procured 
in neuronal culture alone does not fully predict the complex, multi-cellular BDNFinduced output of the slice.

In summary, we have devised a method to traverse an existing gap in extrapolating molecular signaling and translational information from neuronal culture to the events correlated with synaptic plasticity in an intact circuit. BONLAC circumvents the limitations present in existing and frequently used proteomic analysis tools, and together with BONCAT and FUNCAT modifications is specifically suited to dissecting signaling and translational dynamics in three dimensional tissue preparations. Given its short experimental duration, use of readily available reagents, and independence from use of genetically engineered animals, we believe that the technique will allow neuroscientists to address how mature circuits respond to a wide variety of stimuli.

\section{Methods}

Animals

All experiments were carried out using male 10-12 week old male C57/Bl6 mice that were generated in-house or E18 to E19 Sprague-Dawley rat embryos using animal protocols approved by New York University Animal Welfare Committee.

\section{AHA and SILAC Dose}

AHA was synthesized as previously described ${ }^{9}$ and tested for purity using proton NMR for each new batch of synthesis. We tested a range of AHA concentrations from $0.5 \mathrm{mM}$ to $3 \mathrm{mM}$, based on previous reports, and incubated slices with AHA for 1-5 hours after recovery. We found an optimal dose for the application of BONCAT to slices at 1 
mMAHA incubated for three hours (see Fig S1A). SILAC amino acids (13C6-15N2lysine, 13C6-15N4-arginine (Lys8/Arg10) and D4-lysine/13C6-arginine (Lys4/Arg6)) were obtained from Cambridge Isotope Laboratories, MA, USA in concentrations previously described ${ }^{12}$.

\section{Acute hippocampal slice preparation and incubation}

$400 \mu \mathrm{m}$ transverse slices were obtained as previously described ${ }^{45}$. Slices were allowed to recover in ACSF first for $15 \mathrm{~min}$ at room temperature, then at $32^{\circ} \mathrm{C}$ for $45 \mathrm{~min}$. Subsequently, $1 \mathrm{mM}$ AHA (and SILAC amino acids for BONLAC experiments) were introduced in the incubation chamber and slices were incubated for an additional 3-4 hrs depending on whether BONCAT, BONLAC or FUNCAT was utilized. This was followed by treatment with $25 \mathrm{ng} / \mathrm{ml}$ BDNF (Peprotech,NJ, USA) for $1 \mathrm{hr}$. Inhibitors, when applied were added $1 \mathrm{hr}$ in advance of BDNF for BONCAT Western blot experiments. Concentrations of the inhibitors: $25 \mu \mathrm{M}$ of actinomycin D (Tocris, UK), $1 \mu \mathrm{M}$ TTX (Abcam,

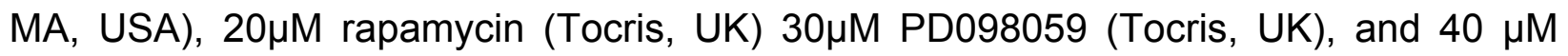
anisomycin (Tocris, UK). Slices then were flash frozen for either mass spectrometry or western blot, or fixed in 4\% PFA for FUNCAT.

\section{BONCAT in hippocampal slices}

Flash frozen sections were thawed and sonicated in lysis buffer ${ }^{45}$ before cycloaddition at room temperature overnight according to the specifications of the manufacturer (Protein Labeling Kit, Invitrogen, CA, USA). Chelating agents such as EDTA were omitted in the lysis buffer because they interfere with the cyclo-addition process. An 
aliquot of the original lysate was retained for BCA protein estimation BCA Kit (Pierce, IL, USA). $30 \mu \mathrm{g}$ of protein from each sample was loaded on $4-12 \%$ Bis-Tris gradient gels (Invitrogen), followed by transfer onto nitrocellulose membranes (GE Healthcare, UK). Membranes were blocked with 3\% BSA and probed with either 1:2000 StreptavidinHRP for $1 \mathrm{hr}$ at room temperature or 1: 2000 anti-biotin (Abcam) overnight at $4^{\circ} \mathrm{C}$. The anti-biotin signal was developed using 1:5000 anti-goat HRP (Jackson Immunoresearch, PA, USA). Blots were developed using either standard or enhanced chemiluminescence detection (GE Healthcare, CT, USA) and imaged using a KODAK 4000MM imaging system. Endogenous proteins labeled with biotin as a co-factor served as a convenient internal loading control for biotin labeling and detection (white arrowsshown in Supplemental Figure 2). These bands did not change with treatment compared to standard loading controls (examples show in Supplemental Figures 2-3). Exposures were set to obtain signals in the linear range and then normalized by total protein and quantified via densitometry. Image adjustments were made for some blots for ease of presentation in printed manuscript, however, blots were quantified without any image manipulation. Other antibodies used for immunoprecipitation (IP) or Western blot (WB) are as follows: Cacna2d1 (Thermo Fisher; 1:250 IP, 1:500WB), Sort-1 (Abcam; 1:2000 IP and WB), Syt-7 (Abcam, 1: 1000 IP, 1:2000 WB), phospho-S6 and total S6 (data not shown) (Cell Signaling Technologies, MA, USA; as described in Bhattacharya et al., 2012), phospho-TrkB (Fig. S2A) ${ }^{43}$ TrkB (Millipore, 1:2000) Actin (Cell Signaling Technologies, 1:10,000).

Flourescent labeling of AHA peptides in hippocampal slices 
To determine the spatial and regional pattern of the translational response in hippocampal slices we used FUNCAT ${ }^{15}$ (fluorescent non-canonical amino acid tagging). Here AHA is detected using an alkyne tagged to Alexa 488 and subsequent fluorescence imaging. At the end of the incubation, slices were transferred immediately to ice-cold $4 \%$ PFA for overnight fixation at $4^{\circ} \mathrm{C}$. The next day, slices were mounted in $3 \%$ agarose and sliced using a vibratome to a thickness of $40 \mu \mathrm{m}$. Free floating sections were collected in PBS and washed twice (5 min each wash). Slices were blocked and permeabilized with a solution of 5\% BSA (Sigma,) $+5 \%$ normal goat serum (Invitrogen) $+0.3 \%$ Triton $\mathrm{X}-100$ in PBS for 90 min with agitation at room temperature. Slices then were washed 3 times with PBS for 5 min each followed by incubation with $500 \mu \mathrm{l}$ of cyclo-addition reaction mix (reagents available as Cell Reaction Kit, Invitrogen) for overnight cyclo-addition at $4^{\circ} \mathrm{C}$ with gentle rocking . Slices then were washed thoroughly in PBS the following day (3 times for 10 min each) and mounted using prolong-Gold antifade reagent containing DAPI (Invitrogen). Confocal imaging was done using an LSM 510 laser-scanning confocal microscope equipped with a 40x Plan Neofluor (NA 1.3) differential interference contrast oil immersion objective (Carl Zeiss Microlmaging, Germany). All images were taken with uniform laser power, gain and offset within a single experiment. All images were subsequently processed using ImageJ ( $\mathrm{NIH}$, USA). For analysis of AHA-Alexa-488 signal, 20 regions of interest (ROI) corresponding to soma size were selected from $40 \mathrm{x}$ brightfield images and were then measured in AHAAlexa-488 images. Arbitrary fluorescent units were taken from the minimum value in the $\mathrm{ROI}$ to preserve the dynamic range as the increase in fluorescence between control and BDNF treatment differed by several folds. 
For determining whether the BDNF-mediated increase in translation was either restricted to specific layers or was uniform throughout the slice. We re-sectioned individual $400 \mu \mathrm{m}$ BDNF-treated slices into thinner $40 \mu \mathrm{m}$ slices in serial order and analyzed every third slice (Supplementary Figure S3B-D). All images were taken with uniform laser power, gain and offset within a single experiment. All images were subsequently processed using ImageJ (NIH, USA). AHA-Alexa-488 signal was quantified first using an average of 3 ROls of 112 pixels $\times 112$ pixels taken from each 10X image of the $40 \mu \mathrm{m}$ slices with background subtracted. The BDNF and control treated sections were then paired so that their brightest outer sections corresponded, and a ratio was taken as average BDNF signal per section over average control signal per section throughout the slice.

Immunoprecipitation of AHA-tagged de novo proteins

Protein lysates tagged with AHA underwent the cycloaddition reaction as described earlier. The lysates were diluted with PBS to a final volume of $1 \mathrm{ml}$ and excess biotin was removed by dialysis against PBS overnight at $4^{\circ} \mathrm{C}$. AHA-biotin-tagged proteins were precipitated using Streptavidin Agarose resin (Thermo Scientific, IL, USA) overnight at RT. Washes and elution were performed as described previously ${ }^{39}$. A mixture of Laemmli buffer and beads was added to the gel and lysates and analyzed by western blot. Supernatant and washes also were run on the blots to verify that biotinlyated proteins were not present (data not shown).

Enrichment of AHA labeled proteins and LC/MS 
BONLAC experiments has a typical time course as shown in Figure 2A. Slices were incubated with AHA and either heavy- or medium-labeled arginine and lysine for four hours and then exposed to BDNF for 1 hour. We chose an extended incubation time for the weighted amino acids to facilitate greater incorporation of these probes into slices. Pilot experiments carried out with incubation times of less than four hours yielded poor MS coverage (data not shown).

Sample Preparation for Mass Spectrometry

Briefly, after labeling and BDNF treatment, cells and slices were lysed in buffer containing $8 \mathrm{M}$ urea, $200 \mathrm{mMTris} \mathrm{pH} 8,4 \%$ CHAPS, $1 \mathrm{M} \mathrm{NaCl}$, and protease inhibitor cocktail (Sigma). Lysates of the samples treated either with or without BDNF were combined in equal volume. The mixed lysate was sonicated to reduce viscosity. AHAlabeled nascent proteins were covalently coupled to alkyne agarose beads using reagents provided in the kit. Beads were transferred to a SDS wash buffer (1\% SDS, $100 \mathrm{mMTris} \mathrm{pH} 8,250 \mathrm{mMNaCl}$ and $5 \mathrm{mM}$ EDTA) and proteins on beads then were reduced with DTT at $70{ }^{\circ} \mathrm{C}$ and alkylated with iodoacetamide at room temperature. The beads then were washed sequentially with 100 column volume of the following three buffers to remove non-specific binding proteins: (1) the SDS wash buffer, (2) $8 \mathrm{M}$ urea, and (3) $20 \%$ acetonitrile. Immobilized proteins were digested with trypsin on-resin at 37 ${ }^{\circ} \mathrm{C}$ overnight in $25 \mathrm{mM}$ ammonium bicarbonate and the resulting tryptic peptides were desalted using StageTips and dried under vacuum in a SpeedVac device.

Liquid Chromatography and Mass Spectrometry 
For LC-MS, a Thermo Scientific EASY-nLC 1000 coupled to a Q Exactive mass spectrometer (Thermo Fisher Scientific) was used. A self packed $75-\mu \mathrm{m} \times 25-\mathrm{cm}$ reverse phase column (Reprosil C18, $3 \mu \mathrm{m}$, Dr. Maisch $\mathrm{GmbH}$, Germany) was used for peptide separation. Peptides were eluted by a gradient of $3-30 \%$ acetonitrile in $0.1 \%$ formic acid over $120 \mathrm{~min}$ (for the slice samples) or $240 \mathrm{~min}$ (for the neuron samples) at a flow rate of $250 \mathrm{~nL} / \mathrm{min}$ at $45^{\circ} \mathrm{C}$. The $\mathrm{Q}$ Exactive was operated in data-dependent mode with survey scans acquired at a resolution of 50,000 at $\mathrm{m} / \mathrm{z} 400$ (transient time $=$ $256 \mathrm{~ms}$ ). Up to the top 10 most abundant precursors from the survey scan were selected with an isolation window of 1.6 Thomsons and fragmented by higher-energy collisional dissociation with normalized collision energies of 27 . The maximum ion injection times for the survey scan and the MS/MS scans were $60 \mathrm{~ms}$, respectively, and the ion target value for both scan modes were set to $1,000,000$.

\section{Protein Identification and Quantitation}

The raw files were processed using the MaxQuant computational proteomics platform version 1.2.7.0 for peptide identification and quantitation. The fragmentation spectra were searched against the Uniprot mouse (for brain samples) or rat (for cultured neuron samples) protein database (both downloaded on Feb 08, 2013), allowing up to two missed tryptic cleavages. Carbamidomethylation of cysteine was set as a fixed modification, and oxidation of methionine and protein $\mathrm{N}$-terminal acetylation, D4-lysine, 13C6-arginine, 13C6-15N2-lysine and 13C6-15N4-arginine were used as variable modifications for the database search. The precursor and fragment mass tolerances were set to 7 and 20 ppm, respectively. Both peptide and protein identifications were filtered at $1 \%$ false discovery rate (FDR). 
All raw mass spectrometry data are deposited at ftp://MSV000078919@massive.ucsd.edu.

To ensure that specific subsets of proteins were not selected artificially, we determined the average fold-change for each protein with no cut-offs and manually classified them into one of ten categories based on their primary role in translation, signaling, transcription, trafficking, protein folding, synaptic function, cytoskeleton, metabolism, and degradation (Supplementary Figure S4A). The protein hits fell into a variety of functional bins, with no apparent bias suggesting that a wide variety of proteins were detected with BONLAC and that the profiling approach in slices were representative of a global proteome.

Computational processing of BONLAC MS data and rank-order consistency score (Cscore)

Raw files were processed using the MaxQuant software package (v. 1.2.7.0 $)^{46}$ with the default parameters, including static modifications (carbamidomethylation at cysteine), and a minimum ratio count of 2 . The main search was run with FASTA sequences obtained from UNIPROT (August 2013 version), and the first search with the mouse.fasta file provided by MaxQuant. A fasta file of common mass spectrometry contaminants was added to the search.

MaxQuant normalized $\mathrm{H} / \mathrm{M}$ ratios (heavy vs. medium isotopes) were used for quantitative analysis. Ratios were inversed for experiments with reversed isotopic labeling. Total intensities were calculated as the sum of the heavy and medium ion intensities, as reported by MaxQuant. The statistical significance of the log-2 transformed ratios and total intensities was analyzed by the Perseus software (v. 
1.1.1.34) using the Significance_B option and the Benjamini-Hochberg correction to account for multiple hypothesis testing. Proteins with $p \leq 0.3$ were selected and then scored for consistency. Only those proteins measured at least twice and those that were consistently up or down regulated each time they were detected were selected. Only those proteins that met all these criteria were deemed candidates. This method provides a clear set of differentially up- or down-regulated proteins (Supplementary Table S1), taking variation between replicates and total ion intensity (signal strength) into account.

\section{Gene Ontology (GO) Analysis}

Gene Ontology analysis was performed using the Database for Annotation, Visualization and Integrated Discovery (DAVID) ${ }^{24}$. Candidate proteins were added as the gene list, and the background was considered as all proteins measured in hippocampal brain slices (Supplementary Table S2). Clusters with enrichment scores over 1.3 with FDR values of less than 0.05 were considered, with the most focus given to candidates that appeared multiple times in clusters that met this criteria.

To complement the functional clustering data obtained from DAVID, we used BiNGO GO software ${ }^{25}$ to map the interactions of the candidate proteins, again finding that a substantial number of the most consistently hits were involved in synaptic release (Figure 2E). Groups with $p<0.05$ were included.

BONLAC in cultured neurons 
Cortical and hippocampal neurons in culture were prepared as previously described from E18 to E19 Sprague-Dawley rat embryos ${ }^{20}$ and grown to DIV10 in Neurobasal supplemented with B27, 37\% glucose and Glutamine (Gibco/Invitrogen, USA). Neurons then were starved of amino acids for 30 minutes, supplemented with media with B27, glucose and glutamine and lacked methionine, arginine and lysine, and then treated with SILAC media and AHA. $25 \mathrm{ng} / \mathrm{ml}$ of either BDNF or vehicle (water) was added with the AHA- and SILAC-containing media after the 30-min amino acid starvation period. Cells were labeled in amino acid free RPMI, supplemented with an amino acid mixture with manually weighed amino acids, except for methionine, arginine and lysine (which were supplemented with AHA and SILAC amino acids), in quantities that were consistent with Neurobasal media (Invitrogen, USA). DIV10 cultured neurons were exposed to either BDNF or vehicle for 2 hours. The two-hour labeling scheme was adapted from earlier BONLAC work in HEK cells ${ }^{12}$ (Figure 4A). After treatment we utilized the same MS and analytical workflow using C-score to select consistently changed candidate proteins.

\section{Acknowledgements}

We would like to thank KatrinDeinhardt, PrakashSubramanyam, Barbara Hempstead, and Francis Lee, as well as the Hempstead lab for valuable input and feedback during the initial design and standardization stages of this work. We also thank Maggie Mamcarz and Sameer Aryal from the Klann lab for technical support. We thank Rebecca Bish for help with the manuscript. This work was carried out with support from the NIH (Training Grant T32 MH019524-20 supported H.B.; NS034007 and NS047384 
to E.K.; NS21072, and HD23315 to M.V.C.; NS050276 and RR027990 to T.A.N.; GM113237 to C.V.). This work also was supported by the Simons Foundation (274864 to E.K.). Support from the NSF is gratefully acknowledged (CHE-1152317 to K.K.). A.B was supported FRAXA Foundation and Charles H. Revson Postdoctoral fellowships during the project period.

\section{References}

1. Costa-Mattioli, M., Sossin, W.S., Klann, E. \&Sonenberg, N. Translational control of long-lasting synaptic plasticity and memory. Neuron. 61(1):10-26. (2009)

2. Richter, J.D.\& Klann, E. Making synaptic plasticity and memory last: mechanisms of translational regulation. Genes Dev. 23(1):1-11. (2009)

3. Hanus, C.\& Schuman, E.M. Proteostasis in complex dendrites. Nat Rev Neurosci. 14(9):638-48. (2013)

4. Schwanhäusser, B., Busse, D., Li, N., Dittmar, G., Schuchhardt, J., Wolf, J., Chen, W.\&Selbach, M. Global quantification of mammalian gene expression control. Nature. 473(7347):337-42. (2011)

5. Liao, L., McClatchy, D.B. \& Yates, J.R. Shotgun proteomics in neuroscience. Neuron. 63(1):12-26. (2009)

6. Dieterich, D.C., Link, J.A., Graumann,J., Tirrell, D.A. \& Schuman, E.M. Selective identification of newly synthesized proteins in mammalian cells using bioorthogonalnoncanonical amino acid tagging (BONCAT). ProcNatlAcadSci USA. 103(25):9482-7. (2006)

7. Ong, S.E., Blagoev, B., Kratchmarova, I., Kristensen, D.B., Steen, H., Pandey, A. \& Mann, M. Stable isotope labeling by amino acids in cell culture, SILAC, as a simple and accurate approach to expression proteomics. Mol Cell Proteomics. 1(5):376-86. (2002)

8. Spellman, D.S., Deinhardt, K., Darie, C.C., Chao, M.V. \&Neubert, T.A. Stable isotopic labeling by amino acids in cultured primary neurons: application to brain-derived neurotrophic factor-dependent phosphotyrosine-associated signaling. $\mathrm{Mol} C \mathrm{Cell}$ Prot7(6):1067-76. (2008) 
9. Link, A.J., Vink, M.K. \&Tirrell, D.A. Synthesis of the functionalizable methionine surrogate azidohomoalanine using Boc-homoserine as precursor. Nat Protoc. 2(8):1884-7. (2007)

10. Howden, A.J., Geoghegan,V., Katsch, K., Efstathiou, G., Bhushan, B., Boutureira, O., Thomas, B., Trudgian, D.C., Kessler, B.M., Dieterich, D.C., Davis, B.G. \&Acuto, O. QuaNCAT: quantitating proteome dynamics in primary cells. Nat Methods. 10(4):343-6. (2013)

11. Selbach, M., Schwanhäusser, B., Thierfelder, N., Fang, Z., Khanin, R. \&Rajewsky, $\mathrm{N}$. Widespread changes in protein synthesis induced by microRNAs. Nature. 455(7209):58-63. (2008).

12. Zhang, G., Bowling, H., Hom, N., Kirschenbaum, K., Klann, E., Chao, M.V. \&Neubert, T.A. In-depth Quantitative Proteomic Analysis of Protein Translation Induced by Brain-derived Neuropathic Factor. Journal of Proteome Research. 13(12):5707-14. (2014)

13. Aakalu, G., Smith, W.B., Nguyen, N., Jiang, C. \& Schuman, E.M. Dynamic visualization of local protein synthesis in hippocampal neurons. Neuron. 30(2):489-502. (2001)

14. Liao,L., Pilotte, J., Xu,T., Wong, C.C., Edelman, G.M., Vanderklish, P. \& Yates, J.R. 3rd. BDNF induces widespread changes in synaptic protein content and up-regulates components of the translation machinery: an analysis using high-throughput proteomics. Journal of Proteome Research.6(3):1059-71. (2007).

15. Dieterich, D.C., Hodas,J.J., Gouzer, G., Shadrin,I.Y., Ngo,J.T., Triller, A., Tirrell, D.A. \& Schuman, E.M. In situ visualization and dynamics of newly synthesized proteins in rat hippocampal neurons.Nat. Neurosci.13(7):897-905. (2010)

16. Figurov, A., Pozzo-Miller, L.D., Olafsson, P., Wang, T.\& Lu, B. Regulation of synaptic responses to high-frequency stimulation and LTP by neurotrophins in the hippocampus. Nature. 381(6584):706-9. (2006).

17. Kang,H. \& Schuman, E.M. Long-lasting neurotrophin-induced enhancement of synaptic transmission in the adult hippocampus. Science. 267(5204):1658-62. (1995).

18. Ying,S.W., Futter,M., Rosenblum,K., Webber,M.J., Hunt,S.P., Bliss, T.V.\&Bramham, C.R. Brain-derived neurotrophic factor induces long-term potentiation in intact adult hippocampus: requirement for ERK activation coupled to CREB and upregulation of Arc synthesis. J. Neurosci. 22(5):1532-40.(2002).

19. Kovalchuk, Y., Hanse, E., Kafitz, K.W.\&Konnerth, A. Postsynaptic Induction of BDNF-Mediated Long-Term Potentiation. Science. 295(5560):1729-34. (2002) 
20. Kramár, E.A., Lin, B., Lin, C.Y., Arai, A.C., Gall, C.M. \& Lynch, G. A novel mechanism for the facilitation of theta-induced long-term potentiation by brain-derived neurotrophic factor. J Neurosci. 24(22):5151-61. (2004)

21. Eichelbaum, K., Winter, M., Berriel Diaz, M., Herzig, S. \&Krijgsveld, J. Selective enrichment of newly synthesized proteins for quantitative secretome analysis. Nat Biotechnol. 30(10):984-90. (2012).

22. Butko, M.T., Savas, J.N., Friedman, B., Delahunty, C., Ebner, F., Yates, J.R. $3^{\text {rd }} \&$ Tsien, R.Y. In vivo quantitative proteomics of somatosensory cortical synapses shows which protein levels are modulated by sensory deprivation. ProcNatlAcadSci $U$ S A. 110(8):E726-35. (2013).

23. Bowling, H., Zhang, G., Bhattacharya, A., Pérez-Cuesta, L.M., Deinhardt, K., Hoeffer, C.A., Neubert, T.A., Gan, W.B., Klann, E. \& Chao, M.V. Antipsychotics activate mTORC1-dependent translation to enhance neuronal morphological complexity. Sci Signal. 14;7(308):ra4. doi: 10.1126/scisignal.2004331. (2014).

24. Huang, D.W., Sherman, B.T. \&Lempicki, R.A. Systematic and integrative analysis of large gene lists using DAVID Bioinformatics Resources. Nature Protoc. 4(1):44-57. (2009).

25. Maere, S., Heymans, K. \& Kuiper, M. BiNGO: a Cytoscape plugin to assess overrepresentation of gene ontology categories in biological networks. Bioinformatics. 21(16):3448-3449. (2005).

26. Nykjaer, A. \&Willnow, T.E. Sortilin: a receptor to regulate neuronal viability and function. Trends Neurosci. 35(4):261-70. (2012).

27. Bacaj, T., Wu, D., Yang, X., Morishita, W., Zhou, P., Xu, W., Malenka, R.C. \&Südhof, T.C. Synaptotagmin-1 and synaptotagmin-7 trigger synchronous and asynchronous phases of neurotransmitter release. Neuron. 80(4):947-59. (2013).

28. Liu, H., Bai, H., Hui, E., Yang, L., Evans, C.S., Wang, Z., Kwon, S.E. \& Chapman, E.R. Synaptotagmin 7 functions as a Ca2+-sensor for synaptic vesicle replenishment. Elife. 3:e01524. (2014).

29. Dolphin, A.C. The a $2 \delta$ subunits of voltage-gated calcium channels.BiochimBiophysActa. 1828(7):1541-9. (2013).

30. Arikkath, J. \& Campbell, K.P. Auxiliary subunits: essential components of the voltage-gated calcium channel complex. CurrOpinNeurobiol. 13(3):298-307. (2003).

31. West, A.E., Pruunsild, P. \&Timmusk, T. Neurotrophins: transcription and translation. HandbExpPharmacol. 220:67-100. (2014) 
32. Takei, N., Inamura, N., Kawamura, M., Namba, H., Hara, K., Yonezawa, K. \&Nawa, $\mathrm{H}$. Brain-derived neurotrophic factor induces mammalian target of rapamycindependent local activation of translation machinery and protein synthesis in neuronal dendrites. J Neurosci. 24(44):9760-9. (2004)

33. Leal, G., Comprido, D. \& Duarte, C.B. BDNF-induced local protein synthesis and synaptic plasticity. Neuropharmacology. doi: 10.1016/j.neuropharm.2013.04.005. (2014).

34. Tang, S.J., Reis, G., Kang, H., Gingras, A.C., Sonenberg, N. \& Schuman, E.M. A rapamycin-sensitive signaling pathway contributes to long-term synaptic plasticity in the hippocampus. ProcNatlAcadSci U S A. 99(1):467-72. (2002).

35. Wang, D.C., Chen, S.S., Lee, Y.C. \& Chen, T.J. Amyloid-beta at sublethal level impairs BDNF-induced arc expression in cortical neurons. NeurosciLett. 398(1-2):7882. (2006).

36. Okuno, H., Akashi, K., Ishii, Y., Yagishita-Kyo, N., Suzuki, K., Nonaka, M., Kawashima, T., Fujii, H., Takemoto-Kimura, S., Abe, M., Natsume, R., Chowdhury, S., Sakimura, K., Worley, P.F. \&Bito, H. Inverse synaptic tagging of inactive synapses via dynamic interaction of Arc/Arg3.1 with CaMKIIß. Cell. 149(4):886-98. (2012).

37. Ji ,Y., Lu, Y., Yang, F., Shen, W., Tang, T.T., Feng, L., Duan, S. \& Lu, B. (2010) Acute and gradual increases in BDNF concentration elicit distinct signaling and functions in neurons. Nat Neurosci. 13(3):302-9. (2010).

38. Genheden, M., Kenney, J.W., Johnston, H.E., Manousopoulou, A., Garbis, S.D., Proud. C.G. BDNF stimulation of protein synthesis in cortical neurons requires the MAP kinase-interacting kinase MNK1. J Neurosci. 35(3):972-84 (2015)

39. Hodas, J.J., Nehring, A., Höche, N., Sweredoski, M.J., Pielot, R., Hess, S., Tirrell, D.A., Dieterich, D.C. \& Schuman, E.M. Dopaminergic modulation of the hippocampal neuropil proteome identified by bioorthogonalnoncanonical amino acid tagging (BONCAT). Proteomics. 12(15-16):2464-76. (2012).

40. Tartaglia,N., Du, J., Tyler, W.J., Neale, E., Pozzo-Miller, L. \& Lu, B. Protein synthesis-dependent and -independent regulation of hippocampal synapses by brainderived neurotrophic factor. J. Biol. Chem.276(40):37585-93. (2001).

41. Hoppa, M.B., Lana, B., Margas, W., Dolphin, A.C. \& Ryan, T.A. a2ס expression sets presynaptic calcium channel abundance and release probability. Nature. 486(7401):122-5. (2012).

42. Chen, Z.Y., leraci, A., Teng, H., Dall, H., Meng, C.X., Herrera, D.G., Nykjaer, A., Hempstead, B.L. \& Lee, F.S. (2005) Sortilin controls intracellular sorting of brain- 
derived neurotrophic factor to the regulated secretory pathway. J Neurosci. 25(26):6156-66. (2005)

43. Jeanneteau, F., Deinhardt, K., Miyoshi, G., Bennett, A.M. \& Chao, M.V. The MAP kinase phosphatase MKP-1 regulates BDNF-induced axon branching. Nat Neurosci. 13(11):1373-9. (2010).

44. Parkhurst, C.N., Yang, G., Ninan, I., Savas, J.N., Yates, J.R. 3rd, Lafaille, J.J., Hempstead, B.L., Littman, D.R. \&Gan, W.B. Microglia promote learning-dependent synapse formation through brain-derived neurotrophic factor. Cell. 155(7):1596-609. (2013).

45. Bhattacharya, A., Kaphzan, H., Alvarez-Dieppa, A.C., Murphy, J.P., Pierre, P. \& Klann, E. Genetic removal of p70 S6 kinase 1 corrects molecular, synaptic, and behavioral phenotypes in fragile X syndrome mice. Neuron. 76(2):325-37. (2012)

46. Cox, J, Mann, M. Quantitative, high-resolution proteomics for data-driven systems biology. Annu Rev Biochem. 80:273-99. (2011). 